received a blood transfusion in the 48 hrs preceding diagnosis. No cases of TRAGI were identified.

Conclusion In our population $83 \%$ of infants diagnosed with NEC had PCV $<42$. Anaemia was associated with increased risk for developing NEC, we did not identify any increased risk relating to blood transfusion. Considering the possible causes for anaemia and refining transfusion thresholds in selected cases may be indicated. Further prospective studies may be helpful to identify potential critical Hct levels.

\section{CRP VALUES CAN DIFFERENTIATE BETWEEN A NORMAL POST-OPERATIVE RESPONSE AND SURGICAL COMPLICATIONS}

doi:10.1136/archdischild-2012-302724.0287

SP Sharif, SR Phelps. Paediatric Surgery, The Royal London Hospital, London, UK

Aims A raised post-operative CRP is a source of great anxiety for surgeons and neonatologists. This study aims to assess the postoperative CRP response in neonates who undergo surgery in order to describe a normal physiological response to surgical trauma. This is compared to post-operative changes in neonates undergoing surgery specifically for NEC and neonates who develop surgical complications.

Methods A retrospective study was performed over a one year period (2010) analyzing consecutive neonates undergoing surgery. Daily WCC, platelet count and C-reactive protein measurements were analyzed on consecutive post-operative days in three distinct subgroups: control, surgical complications group and NEC.

Results There was a total of 81 patients (see Table 1).

Abstract 287 Table 1

\begin{tabular}{lllll}
\hline & No. of Patients & $\begin{array}{l}\text { Median } \\
\text { gestational age }\end{array}$ & $\begin{array}{l}\text { Median time to } \\
\text { surgery (days) }\end{array}$ & $\begin{array}{l}\text { Median birth } \\
\text { weight (grams) }\end{array}$ \\
\hline Control & 53 & 37 & 4 & 2270 \\
Complications & 11 & 35 & 2 & 2500 \\
NEC & 17 & $25+2$ & 23 & 785 \\
\hline
\end{tabular}

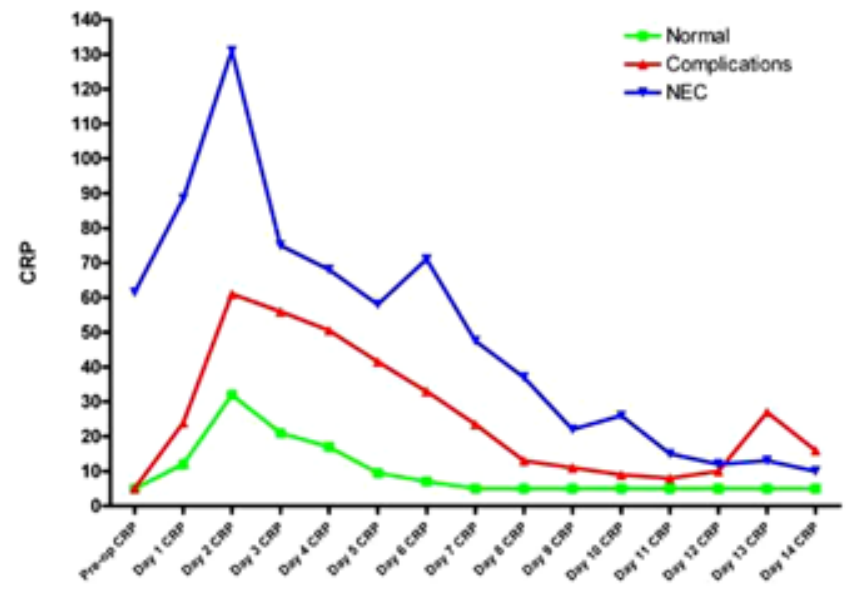

Abstract 287 Graph 1

As shown in Graph 1 the post-operative CRP values for the control group and complications group were significantly different throughout the 12 day time period ( $p=0.01$, Mann-Whitney $U$ test). The CRP values for the control group were also significantly lower than the NEC group ( $p=0.0001$, Mann-Whitney U test).

Conclusion The post-operative CRP trend was more important than a single result. This study quantified a physiological CRP change in response to surgery in neonates. In particular, there was a significantly different CRP response in neonates who developed a post-operative complication.

\section{CLASSIFICATION OF BRAIN INJURY ASSOCIATED WITH NEONATAL ECMO. NATIONAL REVIEW OF 2 DECADES IN THE NETHERLANDS}

doi:10.1136/archdischild-2012-302724.0288

'M Raets, ${ }^{1} \mathrm{~J}$ Dudink, ${ }^{2} \mathrm{H}$ ljsselstijn, ${ }^{3} \mathrm{~A}$ van Heijst, ${ }^{4} \mathrm{M}$ Lequin, ${ }^{2} \mathrm{RJ}$ Houmes, II Reiss, 1.5P Govaert, ${ }^{2} \mathrm{D}$ Tibboel. 'Neonatology; ${ }^{2}$ Intesive Care, Department of Pediatrics and Pediatric Surgery, Sophia Children's Hospital Erasmus Medical Center, Rotterdam; ${ }^{3}$ Neonatology, Radboud University Nijmegen Medical Center, Nijmegen; ${ }^{4}$ Pediatric Radiology, Sophia Children's Hospital Erasmus Medical Center, Rotterdam, The Netherlands; ${ }^{5}$ Pediatrics, Koningin Paola Children's Hospital, Antwerp, Belgium

Background and Aims Brain injury is an important complication of neonatal Extra Corporeal Membrane Oxygenation (ECMO). Unilateral carotid artery and jugular vein cannulation (often with ligation), in combination with systemic heparinisation, increases the risk of brain injury in an already vulnerable group of patient. The reported prevalence of brain injury ranges from $10-52 \%$ of patients treated with neonatal ECMO. Monitoring of intracranial lesions during the ECMO procedure is therefore important for treatment and prediction of outcome.

Our objective is to study incidence and classification of ultrasound proven brain injury during neonatal ECMO in the Netherlands.

Methods Retrospective, nationwide study (Rotterdam and Nijmegen), spanning two decades. Cranial ultrasound images were reviewed by two independent investigators, without knowledge of primary diagnosis, outcome or type of ECMO.

Results 676 neonates with neonatal ECMO were studied. ECMO type was V-A in $88 \%$. Brain abnormalities were detected in $17.3 \%$ of patients: primary hemorrhage was diagnosed most frequent $(8.8 \%)$. A noticeable result was found in stroke patients $(5 \%$ of the total group), where there was a significant predominance of lesions in the left hemisphere. Lobar haematoma (prevalence $2.2 \%$ ) was also significantly left sided predominant.

Conclusion Our study shows an incidence of ECMO-associated neonatal brain injury in the Netherlands in $17.3 \%$. Left hemisphere lesion preference suggests that shift of brain perfusion from right to left is more important than large vessel ligation in the neck. Prevention has to focus on embolism and on management of this perfusion shift.

\section{THE EFFECT OF PERINATAL INFECTION ON NEURODEVELOPMENTAL OUTCOME IN NEWBORNS WITH HYPOXIC-ISCHEMIC ENCEPHALOPATHY}

doi:10.1136/archdischild-2012-302724.0289

${ }^{1} \mathrm{M}$ Jenster, ${ }^{2} \mathrm{~T}$ Ruel, ${ }^{2} \mathrm{SL}$ Bonifacio, ${ }^{2} \mathrm{EE}$ Rogers, ${ }^{3} \mathrm{EW}$ Tam, ${ }^{4} \mathrm{AJ}$ Barkovich, ${ }^{3} \mathrm{DM}$ Ferriero, ${ }^{3} \mathrm{HC}$ Glass. 'University of Groningen, Groningen, The Netherlands; ${ }^{2}$ Pediatrics; ${ }^{3}$ Neurology \& Pediatrics; ${ }^{4}$ Radiology \& Biomedical Imaging, University of California, San Francisco, CA, USA

Studies of preterm neonates suggest that infection may potentiate hypoxic-ischemic (HI) brain injury. In term neonates, infection is a risk factor for encephalopathy and cerebral palsy. Whether it potentiates the risk of brain injury and adverse outcome in the setting of hypoxic-ischemic encephalopathy (HIE) is not clear.

The charts of 257 term newborns with HIE were reviewed for signs of maternal and infant infection, including chorioamnionitis and proven or suspected sepsis. Multivariate logistic regression was used to assess the effect of infection on severity of brain injury as seen on a neonatal MRI (normal-mild vs. moderate-severe), and on risk of adverse neurodevelopment at 30 months in a subset of 
subjects (neuromotor score, NMS $\geq 2$, or Bayley Scales of Infant Development II or III MDI $<70$ or cognitive score $<85$ ).

Chorioamnionitis (42 subjects) was associated with a lower risk of moderate-severe brain injury (OR 0.3; 95\%CI 0.1-0.7; p=0.003), and trended toward lower risk of adverse neurodevelopment. Infant infection (32 subjects) trended toward association with moderatesevere injury (OR 1.6; 95\%CI 0.8-3.5; $\mathrm{p}=0.2$ ), and was significantly associated with an abnormal NMS (OR 3.4; 95\%CI 1.2-10.2; $\mathrm{p}=0.03$ ) but not cognitive outcome. After adjusting for hypothermia and severity of encephalopathy, maternal infection remained associated with a lower risk of brain injury, whereas the association between infant infection and NMS was no longer significant.

These preliminary results are in keeping with animal studies that suggest that the timing of an inflammatory signal may determine whether infection is injurious or protective.

Acknowledgements NIH UL1 RR024131\&P50NS035902.

\section{GENES IMPORTANT IN INFLAMMATION, APOPTOSIS, TRANSCRIPTION REGULATION AND ANGIOGENESIS ARE INDUCED IN THE NEWBORN MOUSE BRAIN AFTER HYPOXIA-REOXYGENATION (HR)}

doi:10.1136/archdischild-2012-302724.0290

1,2AGW Rognlien, 1,2EJ Wollen, ${ }^{2,3} \mathrm{M}$ Atneosen-Åsegg, ${ }^{4} \mathrm{M}$ Bjørås, 1,20D Saugstad. ${ }^{1}$ Department of Pediatric Research, Oslo University Hospital HF; ${ }^{2}$ Women and Children's Division, University of Os/o, Oslo; ${ }^{3}$ Department of Clinical Molecular Biology and Laboratory Sciences, Akershus University Hospital, Lørenskog; ' ${ }^{4}$ Department of Microbiology, Oslo University Hospital HF, Oslo, Norway

Background and Aims Pathogenesis of birth asphyxia has yet to be fully elucidated. To explore the mechanism of HR injury we followed the temporal profile of a priori selected genes in the newborn mouse brain

Methods 84 C57BL/6 mice (postnatal day 7) were randomized to 120 minutes of hypoxia $\left(\mathrm{FiO}_{2} 0.08, \mathrm{n}=64\right)$ or 180 minutes in air (controls (C21), $\mathrm{n}=20$ ). The hypoxia group was randomized to $30 \mathrm{~min}$ reoxygenation with $\mathrm{FiO}_{2} 0.60(\mathrm{H} 60)$ or air $\left(\mathrm{H}_{2} 1\right)$. After observation in air for $0,150,300$ minutes or 3 days, organs were harvested. Homogenate of hippocampus and striatum was analyzed for mRNA expression of 44 genes by real-time PCR.

Results Lcn2, Mt1, Hmox1 and Vegfa were significantly up-regulated $(\mathrm{p}<0.05)$ after $0-300$ min observation when comparing $\mathrm{H}_{2} 1 \mathrm{vsC21}$ and H60vsC21. Ccl2, Ccl12 and Tnf were up-regulated from 0-150 min, Stat3 from 150-300 min, while Ccnd1 was down-regulated at 0 min in both comparisons. In the $\mathrm{H}_{2} 1 \mathrm{vsC} 21$ comparison at 0 min, Neil3 and Apaf1 were down-regulated. When comparing H60vsH21, Cxcl10 (0 min) and Hmox1 (300 min) were up-regulated while Neil3 $(0 \mathrm{~min})$ was down-regulated. There were no significant gene expression changes after 3 days.

Conclusions Genes important in inflammation ( $L \mathrm{cn} 2, \mathrm{Mt1}, \mathrm{Ccl} 2$, Ccl12, Cxcl10, Tnf, Hmox1), apoptosis (Lcn2, Mt1, Tnf, Hmox1, Vegfa), angiogenesis (Vegfa), and transcription regulation (Stat3) were induced up to 300 minutes after hypoxia-reoxygenation while the DNA-glycosylase Neil3 was suppressed. The up-regulation of the pro-inflammatory cytokine Cxcl10 after hyperoxic compared to normoxic reoxygenation, confirms that hyperoxia induces additional inflammation.

\section{1 \\ PROGNOSTIC TESTS IN TERM NEONATES WITH HYPOXIC ISCHEMIC ENCEPHALOPATHY: A SYSTEMATIC REVIEW}

doi:10.1136/archdischild-2012-302724.0291

${ }^{1} \mathrm{H}$ van Laerhoven, ${ }^{2} \mathrm{TR}$ de Haan, ${ }^{3} \mathrm{M}$ Offringa, ${ }^{4} \mathrm{~B}$ Post, ${ }^{5} \mathrm{JH}$ van der Lee. ${ }^{1}$ Academic Medical Center - Emma Children's Hospital; ${ }^{2}$ Neonatology, Academic Medical Center Emma Children's Hospital, Amsterdam, Netherlands Antilles; ${ }^{3}$ Hospital for Sick Children Research Institute, Toronto, ON, Canada; ${ }^{4}$ UMCN St Radboud, Nijmegen; ${ }^{5}$ Woman and Child Centre, Academic Medical Centre, Amsterdam, Netherlands Antilles
Background and Aims Hypoxic Ischemic Encephalopathy (HIE) following perinatal asphyxia in term neonates is associated with long term neurological sequelae or demise. The aim of this systematic review was to investigate the prognostic value of currently used clinical tests in neonatal patients with HIE.

Methods MEDLINE, EMBASE, Central and CINAHL were searched until November 2011. Studies were included if they: (1) concerned infants with a gestational age $\geq 36$ weeks suffering perinatal asphyxia and HIE; (2) evaluated prognostic tests in either cooled or non-cooled patients; (3) reported on neurodevelopmental outcome results at a follow up age $\geq 18$ months. Study selection, assessment of methodological quality, and data extraction was performed by three independent reviewers. Pooled sensitivities and specificities of investigated tests were calculated when possible.

Results Included in the analysis were 29 studies describing 13 different prognostic tests conducted 1631 times in 1306 term neonates. Investigated tests comprised a range of imaging modalities, neurophysiological tests and clinical neurological exams. Most promising neurophysiology tests (first week of life) were: aEEC (sens. 0.93, [95\%CI 0.78-0.98]; spec. 0.90 [0.60-0.98]); EEG (sens. $0.92[0.66-0.99]$; spec. $0.83[0.64-0.93]$ ) and VEP (sens. 0.90 [0.74-0.97]; spec. 0.92 [0.68-0.98]).

Conclusions The available evidence suggests an important role for aEEG, EEG, and VEP. Given the heterogeneity of the tests' performance and outcomes studied, accurate predictions of long term outcomes in these critically ill neonates await the results of well designed large prospective studies that evaluate the best possible combination and timing of diagnostic tests.

\section{THE IMPACT OF HYPOTHERMIA ON POST-NATAL BLOOD BIOMARKERS OF NEONATAL HYPOXIC ISCHAEMIC ENCEPHALOPATHY}

doi:10.1136/archdischild-2012-302724.0292

BH Walsh, GB Boylan, EM Dempsey, DM Murray. Neonatal Brain Research Group, Department of Paediatrics and Child Health, University College Cork, Cork, Ireland

Numerous post-natal biomarkers of hypoxic ischaemic encephalopathy (HIE) severity have been proposed before the era of hypothermia. It is unclear if hypothermia impacts upon these potential biomarkers, and therefore whether previous findings are now valid. The aim of this study was to determine if hypothermia alters the discriminative ability of post-natal nucleated red blood cells (NRBCs) to distinguish between mild and moderate/severely encephalopathic infants.

Methods A prospective cohort study recruited term infants with HIE. The grade of HIE was categorised using Sarnat score, and multi-channel EEG. The recruitment period (2003-2012), spanned the introduction of hypothermia. Therefore the discriminative ability of the NRBC count for grade of encephalopathy could be compared in moderate/severely encephalopathic infants who did and did not receive hypothermia.

Results 86 infants with HIE were included in the study, 40 were mild, 26 moderate (14 normothermic, 12 hypothermic), and 18 severe (10 normothermic, 8 hypothermic). In the normothermic group, the NRBC count discriminated between mild and moderate/ severe Sarnat scores $(p=0.016)$, but not in the hypothermic group $(p=0.297)$. This change was due to a decrease in NRBCs among infants with a moderate Sarnat score receiving hypothermia, This occurred despite these infants having a significantly worse 5 minute Apgar score $(p<0.001)$ and background EEG at 6 hours $(p=0.032)$ than their normothermic counterparts.

Conclusion This study has demonstrated that hypothermia can impact upon early post-natal blood biomarkers of HIE. We therefore advise caution in the use of these samples when studying novel diagnostic biomarkers for HIE in the hypothermic era. 